\title{
Formation des professeurs des écoles en Corse et défi des langues intégrées : voies et problématiques
}

\section{Alain Di Meglio}

\section{QpenEdition \\ Journals}

Édition électronique

URL : http://journals.openedition.org/trema/3227

DOI : $10.4000 /$ trema.3227

ISSN : 2107-0997

Éditeur

Faculté d'Éducation de l'université de Montpellier

Édition imprimée

Date de publication : 1 novembre 2014

Pagination : $102-113$

ISSN : 1167-315X

Référence électronique

Alain Di Meglio, «Formation des professeurs des écoles en Corse et défi des langues intégrées : voies et problématiques», Tréma [En ligne], 42 | 2014, mis en ligne le 16 juin 2015, consulté le 19 avril 2019. URL : http://journals.openedition.org/trema/3227 ; DOI : 10.4000/trema.3227

Ce document a été généré automatiquement le 19 avril 2019

Trema 


\title{
Formation des professeurs des écoles en Corse et défi des langues intégrées: voies et problématiques
}

\author{
Alain Di Meglio
}

1 La mise en place d'un concours spécifique de recrutement des Professeurs des écoles (PE) par la circulaire Lang de 2001 ouvre de nouvelles perspectives pour les langues régionales. Pour la Corse, cette circonstance est concomitante avec le processus dit «Matignon » où la Collectivité Territoriale de Corse voit son rôle renforcé dans le cadre d'une loi où «la langue corse sera normalement enseignée dans le cadre des programmes ».

2 C'est encore dans cette période que l'enseignement d'une langue vivante vient s'inscrire dans les programmes de l'école primaire. Les formations initiale et continue viendront normalement s'inscrire dans ce grand chantier qui a à définir la qualité et la quantité d'enseignement dispensé en langue et pour la langue, cherchant en permanence la distribution idéale entre langue régionale, langue nationale et langue européenne.

Dépasser une vision cloisonnée des langues pour aller vers un enseignement intégré semble la seule voie aujourd'hui. En effet, comment organiser et planifier son enseignement face à ces nouvelles demandes ou injonctions vers l'école? Comment ne pas utiliser le caractère instrumental et transversal des langues dans l'enseignement afin d'éviter un effet cumulatif problématique? Au-delà de ces considérations pratiques, quels avantages y a-t-il à aller vers ce type de système d'éducation bi-plurilingue avec une langue régionale?

4 Pour répondre à ces questions, il convient de bâtir les pratiques et les discours de formation, d'identifier des expériences, de rompre avec des représentations toujours prégnantes et d'avoir les marges de manœuvre nécessaires.

5 Nous proposerons dans notre article une analyse des voies et problématiques liées à ce chantier toujours ouvert à ce jour. 


\section{Quelles langues enseigner à l'école primaire en Corse?}

6 En matière de langues à enseigner, l'académie de Corse se trouve confrontée à un faisceau de logiques ou de contraintes, selon les opinions. En fait, comme dans bien d'autres régions, la conscience territoriale change et vient croiser l'histoire et la géographie pour redistribuer les cartes, remettant en cause la donne de logiques nationales aujourd'hui rendues caduques par les évolutions des territoires politiques. Nous pensons ici à l'avènement de l'Europe, aux effets d'une économie mondialisée ou à une mobilité humaine accrue.

7 Au-delà du français langue première normalement dispensée, l'école en Corse doit répondre à des besoins liés à la langue régionale (le corse) puis à une langue étrangère. Sur ce dernier point, le débat est installé entre une langue européenne utilitaire et dominante, l'anglais et la langue de frontière directe ${ }^{1}$, l'italien, qui jouit par ailleurs d'une forme de reconnaissance de langue historique. Il faut y ajouter l'enseignement des langues et cultures d'origine (ELCO) qui intéressent une population scolaire non négligeable (l'académie de Corse se situant parmi les principales de France en taux de population concernée).

8 Les langues autres que le français sont régies par divers statuts réglementaires et intègrent les programmes selon des cadres fixés au Bulletin Officiel de l'Éducation Nationale (BOEN).

9 Malgré ces cadres bien définis, toute la difficulté réside dans les modalités pratiques et tout particulièrement dans les possibilités d'intégration dans les emplois du temps des écoles primaires déjà bien chargés. Car c'est cette fin de XXe siècle qui voit l'entrée dans les programmes de deux langues (régionale et européenne) alors que le temps des professeurs des écoles devant les élèves s'est réduit passant de 27 heures à 24 heures/ semaine.

10 La langue corse, forte d'une pression revendicative, est aujourd'hui présente sous deux formes distinctes : un enseignement offert (le choix demeurant facultatif) à raison de 3 heures/semaine et un enseignement bilingue à parité horaire (à choisir sous forme de filière) qui partage le temps en langue de façon plus ou moins égale selon les pratiques et les choix des maîtres. Ce statut bilingue existe depuis 2002 grâce à la création d'un concours de recrutement dit « spécifique ».

11 La langue vivante étrangère ${ }^{2}$ (le plus souvent l'anglais) est introduite à partir du CE1 pour un quota annuel de 54 heures (soient en moyenne 1,5 h/semaine). Quant aux ELCO, ils sont dispensés en décrochage à raison d'une heure et demie/semaine.

12 Le professeur des écoles standard ${ }^{3}$ est donc en quelque sorte confronté à de grandes difficultés d'organisation lorsqu'il décide de satisfaire l'ensemble des préconisations officielles : il doit offrir 3 heures d'enseignement de corse, 1,5 heure de langue étrangère et organiser les ELCO.

13 Nos fréquentes visites de terrain et le recueil de témoignages viennent corroborer dans la pratique ces difficultés. Il en découle un ensemble d'arrangements en dehors desquels il est difficile de fonctionner. Par exemple, le temps de 3 heures d'enseignement du corse est théoriquement divisé en deux: une moitié d'enseignements de la langue en tant 
qu'objet d'étude et l'autre réservée en langue régionale à des disciplines non linguistiques (DNL). De même, l'offre des ELCO peut être prise sur du temps périscolaire. Au bout du compte, il apparaît souvent que l'horaire consenti aux DNL en langue corse n'est qu'un affichage, que les ELCO ont du mal à être bien organisés voire offerts et que rarissimes sont les maîtres à pouvoir dispenser de façon autonome la langue régionale et la langue étrangère, ce qui explique aussi de fréquents recours à des intervenants extérieurs.

Ajoutons à cette problématique la complexité des statuts respectifs. Le corse est facultatif pour l'élève mais entre dans le cadre d'une obligation de l'offre par l'école; la langue étrangère est inscrite dans les programmes généraux et la langue d'origine relève d'un droit pour un quota d'élèves issus de l'immigration.

Le constat est évident : l'entrée récente de la diversité linguistique dans l'école primaire remet en cause le principe de contenus disciplinaires communs et pour tous. Le principe est d'autant mis à mal que nous avons évoqué l'existence de filière bilingues français/ corse qui connaissent un certain succès et prennent une place de plus en plus importante grâce à une politique très incitative de la part de la région, en partenariat avec le rectorat de Corse.

16 Au cœur de la logique de socle commun, l'école en Corse introduit par l'enseignement des langues un fait problématique : le choix (de langues et de filière), ce qui peut impliquer une différence dans les approches pédagogiques respectives. Par ces possibilités offertes qui répondent par le choix à une demande de démocratie linguistique, l'école de la République crée une brèche dans le principe des mêmes contenus offerts à tous. Cet état de fait a ouvert, du moins en Corse, le débat sur l'opportunité d'une filière bilingue face à une filière dite "standard", souvent au sein de la même école: faut-il conserver le principe d'un enseignement commun en généralisant le bi-plurilinguisme scolaire sur le modèle des filières bilingues ou bien faut-il instaurer le choix, au motif du respect du caractère facultatif $\mathrm{du}$ corse et des parents non volontaires, mais au risque de trier les publics scolaires (Quenot, 2009) avec les conséquences des effets cachés bien connus des sociologues de l'éducation (choix de filière sur critères ethniques ou d'excellence plus que sur de véritables critères scolaires) ? Pour l'heure, c'est la seconde option qui est retenue.

$17 \mathrm{Au}$ cœur de ces questions vives, la formation des maitres en Corse a donc à tenir compte d'une problématique croisée entre données sociolinguistiques et contraintes pédagogiques. Notre propos ici vise à montrer le rôle joué par les filières bilingues français/langue régionale dans la recherche de solutions à l'introduction des langues à l'école primaire.

\section{La langue régionale comme levier de nouvelles conceptions dans la place et le rôle des langues dans l'école}

18 Dans cette difficulté qui naît clairement du rôle évolutif de l'école publique du XXIe siècle, l'intégration de la langue corse constitue une question socialement vive et vient par là nourrir un débat fécond, tant au plan didactique que sociétal.

Dès 1981 lors de sa création, l'Université de Corse s'est intéressée à cette question. Dix ans après, les premières synthèses vinrent naturellement nourrir la réflexion de la formation des maîtres au sein de l'IUFM qui venait d'être institué. 
20 Ce croisement des thèses entre passion militante, militantisme pédagogique et recherche universitaire a fait éclore une génération de maîtres dont on peut dire qu'ils ont bâti les pratiques didactiques de la langue corse à l'école primaire.

21 Relayés par l'encadrement des conseillers pédagogiques et l'élaboration d'outils ${ }^{4}$ sans cesse en progrès, ils ont su, peu à peu, se forger une praxis en lien étroit avec le discours universitaire. Pour nombre d'entre eux, conscients des potentialités offertes par l'usage des langues en tant qu'outil d'apprentissage, ils se sont ouverts au plurilinguisme scolaire.

\section{1. Des concepts au service du discours des formateurs}

« Nous appelons langue corse la somme de tous les parlers, distingués entre eux par de minces variantes, qui sont utilisés sur le territoire de l'île de Corse. [...] nous rejetons l'idée préconçue d'une clarification par réduction à des formes idéales " (Marchetti/ Geronimi, 1971). Ce précepte est celui de l'ouvrage qui posa les bases de l'orthographe du corse moderne ${ }^{5}$.

23 C'est l'avènement d'une plurinorme (ou norme plurielle) qui accompagnera l'entrée officielle du corse dans l'école en 1974. Tant du côté des usagers de l'écriture que des enseignants, les préconisations orthographiques, présentant un caractère très différent de la langue française, seront très majoritairement adoptées. En l'absence d'une norme officielle et prescrite, le corse déclaré langue proposera une variation graphique certes limitée mais toutefois suffisante pour permettre le respect des principales variétés de corse. Le professeur Fernand Ettori (1981, p. 30), ayant noté l'évolution de la graphie vers cette norme plurielle mais codifiée de façon globalement cohérente et ayant tout autant perçu les avantages à tirer du fait de «n'avoir jamais subi la réduction appauvrissante du bon usage », parlera plus tard de «dialectique de l'un et du multiple qui est celle de la vie » à propos du corse.

Quelques années après, relayée par la recherche universitaire, la formation continue et initiale des enseignants va s'appuyer sur le concept de langue polynomique pour élaborer un ensemble de stratégies qui cherchera les moyens didactiques de refléter le processus naturel de l'évolution du patrimoine linguistique. C'est le linguiste Jean-Baptiste Marcellesi qui propose pour la première fois le concept en 1983 et qui le définit ainsi : «Langues dont l'unité est abstraite et résulte d'un mouvement dialectique et non de la simple ossification d'une norme unique, et dont l'existence est fondée sur la décision massive de ceux qui la parlent de lui donner un nom particulier et de la déclarer autonome des autres langues reconnues ». Allant dans le sens de la tolérance linguistique, la polynomie renforce dans l'enseignement « les pratiques didactiques favorables à la diversité » (Thiers, 2008).

Ces quelques étapes jalonneront le mouvement vers de nouvelles approches didactiques et nourriront le concept de façon impliquée (notamment par un engagement pédagogique) et appliquée (par les riches expériences engendrées). Donnant un bilan de quinze années de formation initiale à l'IUFM de Corse, Jean-Marie Comiti (201, pp. 89-90) écrit à propos de cette période d'application et d'interprétation de cet appareil théorique: «La recherche action prenait vie dans les établissements scolaires où les cohortes d'enseignants auxiliaires, fraîchement diplômés, mettaient à l'épreuve les théories universitaires. Un tâtonnement scientifique qui donnera lieu à une réflexion pédagogique innovante et féconde. » 
26 Il ajoute que dans la formation des maîtres «l'accent est mis [...] sur une éducation induite par la polynomie : reconnaissance et légitimation de la différence inter-dialectale, acceptation de l'Autre dans ses spécificités expressives, dédramatisation des phénomènes interlinguistiques. » On comprend alors toute la fécondité du concept appliqué et les perspectives ouvertes en matière de plurilinguisme.

27 L'approche polynomique de la langue corse a eu pour effet de présenter une alternative à la classe monolingue, modélisée par de longues années de pratiques scolaires issues pour la plupart de la IIIe République. On peut dire sans ambages que pour la Corse, c'est l'introduction de la langue régionale qui ouvre le chantier des savoirs et savoir-faire didactiques de l'éducation scolaire bi-plurilingue.

\section{2. Des caractères pour un modèle qui se cherche}

28 L'approche polynomique a permis le pari improbable de faire entrer dans l'école une langue dont la norme n'avait pas le caractère monolithique des langues didactisées par de longues années d'expérience.

29 Pour la première fois, la salle de classe s'écartait du modèle de l'enseignement d'une seule langue pour se tourner vers une pratique et une reconnaissance ouvertes à la pluralité linguistique : tout d'abord par l'introduction d'un second code enseigné, puis par le fait que ce second code était abordé dans des conceptions normatives plus souples, plus conformes en somme à la réalité langagière du contexte scolaire.

30 Il ne s'agit pas ici de présenter le système de l'éducation bilingue français/corse: il demeure peu harmonisé mais vaut par le regard que l'on peut poser sur un ensemble de pratiques innovantes. Ces expériences posent toutefois la qualité d'une circulation entre la recherche universitaire, l'institut de formation des maîtres et l'élaboration d'une praxis. En 2004, à la suite d'une série d'observations dans les sites bilingues des écoles primaires en Corse, nous constations avec Claude Cortier (Cortier/Di Meglio, p.193) : « Induites, construites ou intégrées à partir d'une formation didactique, les pratiques des sites bilingues en Corse apparaissent comme la résultante d'une situation sociolinguistique qui débouche sur un projet d'école. »

31 Parmi les caractères qui semblent profiler un modèle d'enseignement des langues intégrées, nous avons retenu ici deux critères à l'appui de deux expériences de classe qui nous semblent significatifs.

\section{2. 1. Une polynomie impliquée et appliquée}

Le principe de polynomie a eu pour conséquence de construire la mise en commun de la discipline Langue et Culture Corses sur le respect de la variation linguistique tout en fondant une unité. Cette approche de la continuité linguistique (approche variationniste) a eu pour effet un autre rapport aux langues collatérales : la première, l'italien, s'est retrouvée dans les classes non plus comme langue étrangère et plus largement, c'est l'aire romane qui retrouve un espace de valorisation et qui est le plus souvent objet d'échanges (voyages, visites,..).

33 L'exemple d'une séance observée dans la classe (3 niveaux) du village de Santa Riparata di Balagna nous paraît illustrer parfaitement la maitrise une conduite de type plurilingue. 
L'objectif de la séance est la lecture-compréhension de l'histoire de Pinocchio en corse, français et italien ainsi que la connaissance de l'auteur et de son œuvre. Les élèves sont répartis en petits groupes hétérogènes (différences d'âges et de niveaux). L'activité est organisée de telle sorte qu'elle implique la participation et la motivation de tous et ne peut se dérouler sans l'apport de chacun des groupes. Dans un premier temps, chaque groupe reçoit des bandes de texte dans les trois langues et interviendra en fonction du déroulement de l'histoire et de l'interaction globale. Chaque partie identifiée et lue est affichée au tableau sur lequel se construit donc progressivement un texte plurilingue. Il convient d'insister sur ce dernier point : un seul texte en trois langues. Ici le sens unitaire prime et le plurilinguisme en est l'outil linguistique.

L'analyse des interactions de classe ${ }^{6}$ montre comment l'approche de l'enseignant " casse " la représentation uniciste de la norme linguistique à l'école et encourage la production verbale dans l'apprentissage d'une langue proche comme l'italien.

\section{2. 2. Éveil aux langues et interculturalité}

36 Eu égard à son économie, la démographie scolaire en Corse est plurielle. Les principales communautés sont représentées par les Marocains et les Portugais. Arabe, Amazighe, Portugais et autres langues présentes dans les classes peuvent faire l'objet de valorisation par l'exploitation de contes, chants ou autres activités d'éveil aux cultures et langues. Par des activités de création et par la présence de maîtres de langue d'origine, des productions croisées, interculturelles sont pratiquées.

Chez nombre d'enseignants bilingues expérimentés du premier degré, le repli sur soi autant que le modèle de la langue unique trouvent un dépassement que l'on peut attester par l'observation de choix didactiques et/ou par la lecture de travaux divers (comptes rendus, projets, rapports ou mémoires de conseillers pédagogiques, stages,...). Par le croisement d'aspects axiologiques et praxéologiques se dégage le projet d'une éducation bilingue tendant vers le plurilinguisme. Le modèle recherché oriente alors le creuset de l'école publique vers l'interculturalité et la diversité, en tentant le pari de l'effacement des hiérarchies culturelles.

Nous prendrons ici l'exemple de ML, professeure des écoles habilitée pour l'enseignement bilingue. Elle présente ainsi le lien qu'elle fait entre introduction du corse comme langue seconde et intégration d'élève d'origine étrangère: "La réussite va aussi vers les populations étrangères. Je pense que pour elles, vraiment, c'est un plus, pour les enfants qui vivent ici, qui ne sont pas français, parce qu'ils savent où ils sont, qu'il $\mathrm{y}$ a une autre langue, que ce ne sont pas les seuls à avoir, par exemple, deux langues. Et ils le disent. Ils disent, moi, à la maison, je parle arabe, je parle portugais. » ${ }^{7}$

Nous pouvons illustrer la démarche par deux exemples toujours issus des visites de terrain que nous avons à effectuer. Le premier s'est déroulé dans un cours préparatoire bilingue de l'école Sandreschi de Corte. Le projet a consisté en la réalisation d'une pièce plurilingue, L'œuf $d u$ coq, d'après un conte de Hubert Ben Kemoun ${ }^{8}$ : un coq borgne et maniaque voudrait qu'au zoo, on ne parle plus qu'en " pur français ». Cet animal, entouré d'individus d'autres espèces, va fustiger chez eux le recours à des mots français nés d'emprunts. Au-delà de la valeur de l'histoire qui fustige l'intolérance et le purisme, l'enseignante en a profité pour créer une pièce où les langues incriminées vont être parlées par différents animaux : on débouche ainsi sur un texte en français, corse, arabe et espagnol. Le français et le corse ont été travaillés tous ensemble, l'arabe avec 
l'enseignant de LCO, l'espagnol avec une maman professeure et deux enfants maîtrisant cette langue. On obtient donc un ensemble où l'élève peut mesurer la relativité du signe linguistique et la diversité des codes, qui n'est que celle du capital multilingue de la classe. L'enseignante insiste bien sur le fait que le conte a été choisi en fonction de son intérêt pour une éducation bi-plurilingue. Il s'agit bien ici d'éducation et non de simple apprentissage dans la mesure où les aspects civiques et interculturels sont recherchés.

Autre exemple, à l'école de Cardo (Bastia), cette fois. Le « Villayet » est une bâtisse érigée à la fin du XIXe siècle par un ingénieur qui travaillait à la construction du canal de Suez. Son architecture est le fruit d'un mélange d'influences : orientale, suisse, corse,... Le projet de pièce de théatre "Cumedia à $\mathfrak{u}$ Villayet " a été inspiré par ce lieu très particulier qui a frappé l'imagination de l'enseignante qui l'a relié à un autre récit publié par le CRDP de Corse, Saveriu è i Turchi ${ }^{9}$.

L'équipe pédagogique a proposé un récit à ses élèves à partir de photographies réalisées par un professionnel : les Sarrazins qui vivaient dans le Villayet résistaient aux Corses qui voulaient le reprendre. À la fin, le mariage de la fille du sultan avec le fils du berger est scellé par un échange de secrets : celui du brocciu pour les Corses et celui du fer pour les Sarrazins. L'élaboration de la pièce s'est faite en commun et s'écrivit naturellement sur une alternance plurilingue.

\section{2. 3. Un maître deux langues (et plus) en immersion partielle}

Dans un format assez régulier, ces exemples affirment aussi un modèle résolument tourné vers une alternance des codes pratiquée par le même maître. Mattea Lacave (2011, p.9) dans son mémoire de Master qui fait le point sur sa pratique de professeur des écoles bilingue déclare: "L'immersion, quand elle ne descend pas en-dessous de $50 \%$ est intéressante. Elle enlève, à l'apprentissage des langues, le caractère uniquement disciplinaire de matière à enseigner, qu'on tendait à lui conférer ». Elle adosse par ailleurs son propos sur des préceptes fondés sur une approche communicative de la didactique des langues : « Pour Laurent Gajo ${ }^{10}$ :

"l'immersion appartient aux méthodes communicatives. Dans la mesure où elle privilégie, dans un premier temps, le sens sur la structure et parce que la grammaire n'est pas enseignée, dans les deux langues, à coups de batteries d'exercices». C'est un enseignement implicite, ludique et basé sur la communication [...]» (ibid.)

Ce parti pris de l'enseignant unique bi-plurilingue marque bien une volonté d'intégration, de continuité de l'enseignement sans décrochage de groupe et sans cloisonnement des codes. Il recherche ici clairement une unité d'enseignement pour tous et de façon commune. Nous analysons ce fait comme une forme syncrétique des valeurs de l'enseignement public du premier degré en France et d'une volonté nouvelle de dépasser un monolinguisme scolaire que le contexte local et macropolitique rend caduc. Dans la continuité des valeurs de l'école primaire, on constate en effet une volonté intacte d'aller vers une mise en commun et un partage du savoir sans cloisonnement. L'effet alternatif se situe dans le fait que c'est le divers qui se substitue à l'unique en termes d'approches linguistiques et culturelles. Même si d'autres approches peuvent amener à ce type d'expérience (éveil aux langues, approches interculturelles,...), nous postulons que c'est l'effet de l'introduction de la langue corse conceptualisée par la polynomie qui a conduit à ces nouvelles pratiques. 


\section{Un chantier toujours ouvert}

Notre collègue Claude Cortier, à la suite d'une série d'observations en Corse et ailleurs arrivait à la conclusion suivante: "L'ouverture de nos travaux à d'autres régions de France où se développe une éducation bilingue (Catalogne nord, Occitanie) nous a permis de retrouver des orientations similaires dans l'organisation didactique et dans les valeurs et principes, sans pour autant que la réflexion soit aussi avancée et les principes structurés et systématisés.

La valorisation de ces pratiques et des enseignants qui les mettent en œuvre nous apparaît comme une priorité actuelle, dans la mesure où ces initiatives concernent des domaines essentiels à la cohésion sociale et à la citoyenneté européenne (intégrant également les rapports Nord/Sud).» (Cortier/Di Meglio, 2008, p. 206)

Nous pouvons résumer les exigences du système scolaire public en Corse en quatre points fondamentaux: assurer l'unité de la nation en cultivant son sentiment; sauver et promouvoir les langues régionales (en Corse, statut de la LCC); respecter la diversité identitaire (ELCO) ; construire l'Europe (enseigner une langue étrangère).

La réponse de la recherche en didactique des langues en Corse que relaie le discours de la formation des maitres (et que l'on retrouve sous forme de pratique et de praxis auprès d'un certain nombre de maîtres) va clairement vers une préconisation de fonctionnement intégré des langues. Il pourrait se décliner ainsi sur ces quatre critères :

- didactique intégrée des langues ;

- intercompréhension entre langues parentes, collatérales ;

- approches interculturelles ;

- approches plurielles des langues et cultures.

Les mémoires des étudiants se destinant à l'enseignement bilingue français/corse témoignent globalement de la prise en compte des préceptes qui les conduisent vers la démarche intégrée évoquée ici.

Pour autant les formations initiale et continue ont du mal à dépasser le cadre du bilinguisme langue nationale/langue régionale pour forger une véritable dimension biplurilingue. On peut attribuer cette carence à plusieurs raisons :

- les langues étrangères hésitent fortement à se départir de leur propre cadre didactique pour favoriser une approche de langue voisine ;

- l'IUFM (aujourd'hui ESPE ${ }^{11}$ ) de Corse, malgré de réelles avancées dans la préparation/ formation au concours spécifique, n'a pas pour l'heure réussi le pari de ce partage avec les professeurs de langue étrangère ;

- l'approche intégrée des langues demande une formation des formateurs que l'IUFM de Corse n'a pas eu le temps de mettre en place ;

- on peut dire qu'en une trentaine d'années, la langue corse a pu s'ériger en discipline scolaire grâce aux effets coalisés de l'expérience de terrain, de la formation relayée par la publication d'outils didactiques par le CRDP de Corse. Pour l'heure, les riches expériences évoquées dans cet article n'ont pas réussi à être fédérées par la même dynamique et 
suffisamment diffusées par une politique de stage et par la production d'outils (issus de ces potentiels travaux de stages).

La didactique du bi-plurilinguisme en Corse reste donc à bâtir formellement ;

- au-delà de la formation initiale, le suivi des professeurs bilingues s'avère insuffisant par la suite. Il a par ailleurs du mal à s'harmoniser avec les pratiques d'autres professeurs bilingues (non issus du même système de formation pour diverses raisons de parcours de carrière), ce qui pose le problème d'une formation continue renforcée et efficace dans ce secteur encore en chantier. Or, on ne peut que déplorer la baisse actuelle de l'offre de formation continue, faute de brigades de remplacement.

52 C'est pourquoi il convient de relativiser les avancées que nous pouvons constater parmi les professeurs des écoles bilingues de Corse. Lors de récentes réflexions sur les évolutions du bilinguisme scolaire depuis l'avènement du concours spécifique en Corse, nous constations que «si l'année 2002 marque une ère nouvelle pour l'enseignement du corse, il reste cependant à mesurer l'impact d'une relative massification des enseignants, des apprenants, des parents usagers et de l'encadrement. Le phénomène est récent mais il change fondamentalement la donne socio-éducative en passant d'une somme d'expériences avancées, fortement motivées et souvent relayées par l'Université à une généralisation en cours difficile à maîtriser en terme d'harmonisation des objectifs et des pratiques. » (Di Meglio/Quenot, 2010).

53 Il $\mathrm{y}$ a donc à constater un chantier passionnant mis en place par la recherche en didactique des langues, la formation des maîtres en institut universitaire et les pratiques des maîtres bilingues ou PEMF les plus expérimentés en ce domaine.

Il répond sans ambages à une demande politique et sociétale tout en militant pour une nouvelle démocratie linguistique que l'Europe et beaucoup de régions appellent de leurs vœux.

Le défi est de taille et se heurte sans doute à des problématiques idéologiques, mais il révèle encore nombre d'obstacles conceptuels, techniques et pratiques.

Il ressort de l'expérience corse que la solution ne réside pas dans une vision cloisonnée et superposée des langues à introduire dans les cursus scolaires mais qu'elle passe par une approche intégrée et dans une gestion intelligente de l'immersion partielle (de type Emile ${ }^{12}$ en Europe par exemple).

57 L'option polynomiste choisie par les sites bilingues en Corse, utile dans les conceptions et représentations sociolinguistiques, a su préparer une partie des enseignants à une interprétation régionale d'un plurilinguisme corrélé à des solutions pédagogiques et proches des préconisations du Cadre Européen Commun de Référence pour les Langues.

\section{BIBLIOGRAPHIE}

Comiti, J.-M. (2012). La polynomie dans le discours de la formation de formateurs en langue corse. In Ottavi, P. (Dir.), La langue corse dans le système éducatif. Enjeux sociaux, curriculaires et didactiques du bi/pluriliguisme, Ajaccio, Albiana/Université de Corse. 
Cortier C. \& Di Meglio A. (2012). Le dépassement du conflit diglossique en Corse : implications pédagogiques et didactiques chez les maîtres bilingues de l'école primaire. In Ducancel, G. \& Lee Simon, D. (Ed.). Revue INRP- Repères $n^{\circ} 29$ / 2004, Français et langues étrangères et régionales à l'école, quelles interactions ? Consultable en ligne : http://ife.ens-lyon.fr/publications/editionelectronique/reperes/RS029-10.pdf (lu 27/07/2012)

Cortier C. \& Di Meglio A. (2007). Pédagogie interactive, gestion plurilingue et rapport à la norme dans les écoles bilingues français-corse. In Auzanneau, M. (Ed.), La mise en œuvre des langues dans l'interaction, Paris, L'Harmattan.

Cortier C. \& Di Meglio A. (2008). L'éducation bi-/plurilingue en milieu scolaire corse. In Budach, G. ; Erfurt, J. ; Kunkel, M. (Ed.), Écoles plurilingues - multilingual shools : konzepte, Institutionen und Akteure, Peter Lang ed, Frankfurt.

Di Meglio A. \& Quenot S. (2010). Les choix problématiques de l'enseignement bilingue en corse. In Toreilles, C. \& Verny, M.-J. (Ed.), Enseigner une langue régionale, revue Les langues modernes de l'association APLV, $\mathrm{n}^{\circ} 4,2010$. Article en ligne : http://www.aplv-languesmodernes.org/IMG/ pdf/4_2010_di_meglio-quenot.pdf (lu le 30/04/2013)

Ettori F. \& Fusina J. (1981). Langue corse, incertitudes et paris, Ajaccio, Scola corsa et Maison de la Culture de la Corse.

Lacave M. (2004). Enseigner/apprendre deux langues en site bilingue français/corse : une démarche unique en grande section maternelle. (Mémoire professionnel pour le CAFIPEMF). Bastia.

Lacave M. (2011). L'attelli di pratica teatrale cum'è aiutu à a didattica di e lingue è à l'educazione bislingua. (Mémoire de M2). IUFM/Université de Corse.

Marcellesi J.-B. et al. (2003). Sociolinguistique, épistémologie, Langues régionales, Polynomie. Paris : L'Harmattan.

Marchetti P. \& Geronimi D. A. (1971). Intricciate è cambiarine, manuel pratique d'orthographe et d'orthoepie corse. Nogent-sur-Marne : Ed. Beaulieu.

Quenot S. (2009). Curriculum et enjeux sociétaux de l'enseignement bilingue français-corse. In Gonçalves, C. \& Groux, D. (Dir.), Approches comparées de l'enseignement des langues et de la formation des enseignants. Paris : L'Harmattan.

Thiers J. (2008). Papiers d'identité(s). Edition revue et actualisée. Ajaccio : Albiana.

\section{NOTES}

1. Il faut préciser ici que, contrairement à l'Alsace qui a choisi l'allemand standard comme langue régionale, le corse s'est déclaré langue régionale autonome de l'italien, même si la proximité linguistique est reconnue et acceptée comme une évidence géographique et historique.

2. Selon les programmes de 2007 sont concernées « 8 langues : allemand, anglais, arabe, chinois, espagnol, italien, portugais et russe. À l'issue de l'école primaire, les élèves doivent avoir atteint le premier niveau du CECRL, le niveau "A1". Ils sont alors capables de communiquer de façon simple, si l'interlocuteur parle lentement et distinctement. Pour les cycles 2 et 3 , l'enseignement des langues se déroule sur 54 heures annuelles. »

3. La Corse, comme les autres régions concernées en France, distingue le maitre standard (recruté par la voie non spécifique du concours de recrutement des professeurs des écoles) du maître bilingue. Ce dernier est titulaire d'un concours spécifique ou d'une habilitation délivrée 
par un jury académique et il enseigne en corse et en français à parité horaire. Cela précisé, les filières bilingues ne sont pas moins exemptes d'enseignement de la langue étrangère et d'ELCO.

4. Nous pensons notamment ici aux publications du Centre Régional de Documentation pédagogique de Corse qui, avec le soutien de l'institution régionale a su proposer aux enseignants une somme importantes d'outils pédagogiques et didactiques en faveur de la LCC (langue et culture régionales).

5. Les nouvelles orientations orthographiques de Marchetti et Geronimi auront le mérite, à partir du bilan du corse écrit jusqu'en 1971, de proposer, sans volonté d' "édicter un code, personnel, impérieux et comminatoire (...) ni davantage chercher de bouleverser l'usage », une codification cohérente du corse donnant la possibilité d'écrire dans différentes variétés dialectales de l'île.

6. On peut retrouver la présentation et l'analyse complète de cette séance in Cortier/Di Meglio (2007)

7. Propos recueilli lors d'une enquête de terrain à l'école maternelle bilingue Campanari, Bastia (archives personnelles)

8. Ben Kemoun (Hubert), L'œuf du coq, Paris, Duculot, 2005. Présentation de l'éditeur : « Un beau jour arrive au zoo un coq borgne. Il a la prétention d'inculquer aux autres animaux le «pur français». Du coup, sont mis à l'index tous les mots ayant une origine étrangère : maboul, clown, oranges, bananes, café, puma, coyote, gazelle, girafe, chimpanzé, chocolat, scandale. »

9. Alessandri (Jean), Saveriu è i Turchi, CRDP de Corse, 2002

10. Le mémoire cite: Gajo (Laurent), Immersion, bilinguisme et interaction en classe, Paris, Didier, 2001, sans en préciser la page.

11. École Supérieure du Professorat et de l'Éducation

12. Enseignement de Matières par l'Intégration d'une Langue Étrangère

\section{RÉSUMÉS}

L'ouverture d'un concours spécifique de recrutement des Professeurs des écoles (PE) par la circulaire Lang de 2001 ouvre de nouvelles perspectives pour les langues régionales. Pour la Corse, cette circonstance est concomitante avec le processus dit «Matignon » où la Collectivité Territoriale de Corse voit son rôle renforcé dans le cadre d'une loi où «la langue corse sera normalement enseignée dans le cadre des programmes». C'est encore dans cette période que l'enseignement d'une langue vivante vient s'inscrire dans les programmes de l'école primaire. Les formations initiale et continue viendront normalement s'inscrire dans ce grand chantier qui a à définir la qualité et la quantité d'enseignement dispensé en langue et pour la langue, cherchant en permanence la distribution idéale entre langue régionale, langue nationale et langue européenne. Dépasser une vision cloisonnée des langues pour aller vers un enseignement intégré semble la seule voie aujourd'hui. Encore faut-il bâtir les pratiques et les discours de formation, identifier des expériences, rompre avec des représentations toujours prégnantes et avoir les marges de manœuvre nécessaires. Nous proposerons dans notre article une analyse des voies et problématiques liées à ce chantier toujours ouvert à ce jour.

The opening of a specific and competitive recruitment of schools teachers defined by the circular from the Lang Minister in 2001 opens up new prospects for minority (regional) languages. Concerning Corsica, this circumstance is simultaneous with the process said "Matignon" where the Territorial Collectivity of Corsica was strengthened through a law where "the Corsican 
language is normally taught in the programs". It is also in this period that the teaching of a foreign language is part of primary school programs. The initial and in-service teacher training will normally be part of this major project which defines the quality and quantity of teaching language and the language (an ideal distribution between regional language, national language and European languages). Beyond a partitioned view of language, going towards an integrated education seems the only way today. However, teacher training practices need to be built, experiences need to be identified, representations need to be changed and more room for change needs to be given. In this paper, we will propose an analysis of the ways and issues related to this ongoing matter.

INDEX

Mots-clés : Corse, école primaire, formation des maîtres, langues

Keywords : Corsica, elementary school, language, teacher training

\section{AUTEUR}

ALAIN DI MEGLIO

Professeur, Université de Corse, UMR 6240 LISA 